\title{
Random Cocked Hats
}

\author{
Ian Cook
}

(University of Essex)

In his article' on 'The Cocked Hat', J. E. D. Williams gives the value $1 / 4$ for the probability that the true position lies inside a cocked hat. $\mathrm{He}$ also considers the probabilities that the true position lies in the regions outside the cocked hat. The cocked hat gives three 'edge-regions', obtained by crossing one edge of the cocked hat from the interior, and three 'vertex-regions', obtained by crossing through a vertex from the interior. Mr Williams claims (see his Fig. 4, reproduced here as Fig. I) that the probability associated with each edge-region is $I / 6$ and with each vertex-region is $1 / 12$.

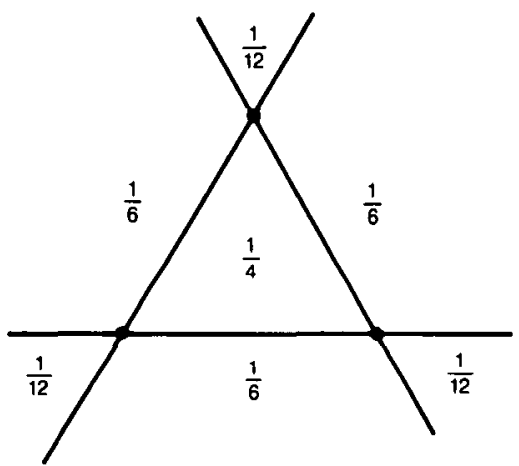

Fig. 1 .

The only assumptions are independence of errors and that each position line is equally likely to pass on either side of the true position. No further assumptions are made as to the actual distributions of the errors. I shall demonstrate by considering some special distributions that the values for the edge-regions and the vertex-regions are incorrect and that these probabilities must depend on the actual distributions of the errors. The value for the probability that the true position is inside the cocked hat is, of course, correct and independent of the distributions of errors.

For definiteness, the three bearing stations are labelled as $\mathrm{P}, \mathrm{Q}, \mathrm{R}$ and the corresponding bearing lines as $p, q, r$. The edge-region on the opposite side of $p$ to the cocked hat will be referred to as the ' $p$-edge region', and similarly for $q$ and $r$. The vertex-region at the vertex where $q$ and $r$ intersect will be referred to as the ' $q r$-vertex region', and similarly for $\mathrm{pq}$ and $\mathrm{pr}$.

Suppose that $P Q R$ is an equilateral triangle with the true position at its centroid and consider the following special distributions of errors. The error in the line $p$ is either $+\alpha$ or $-\alpha$, with equal probability of $\mathrm{I} / 2$. Similarly for $q$ with errors of $\pm \beta$ and $r$ with errors of $\pm \gamma$.

If $\alpha$ and $\beta$ are equal and small, and $\gamma$ is large, then the true position will be close to the $p, q$ vertex and far from $r$. The true position must therefore lie in either the cocked hat or the pq-vertex region, or the p-edge region, or the $q$-edge region, each with 
probability $1 / 4$, since each configuration has probability $1 / 8$. In this case two vertex regions and one edge region are impossible. This is illustrated in Fig. 2, which shows the four possible configurations of $\mathrm{p}$ and $\mathrm{q}$ and in each case the two possible positions of $r$, labelled $r_{1}$ and $r_{2}$. The true position is marked by a cross.
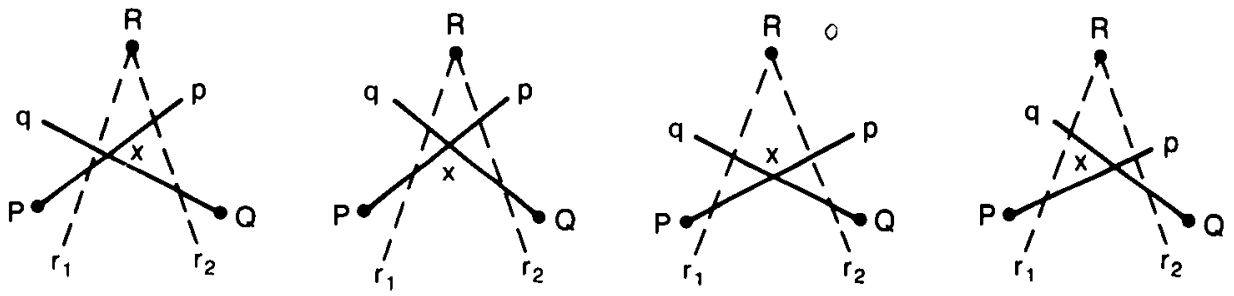

Fig. 2.

If $\alpha$ and $\beta$ are equal and large, and $\gamma$ is small, the true position will be close to $r$ and the possible configurations are shown in Fig. 3. We see that, in this case, all three vertex regions are impossible and that the three edge regions each have probability $1 / 4$.
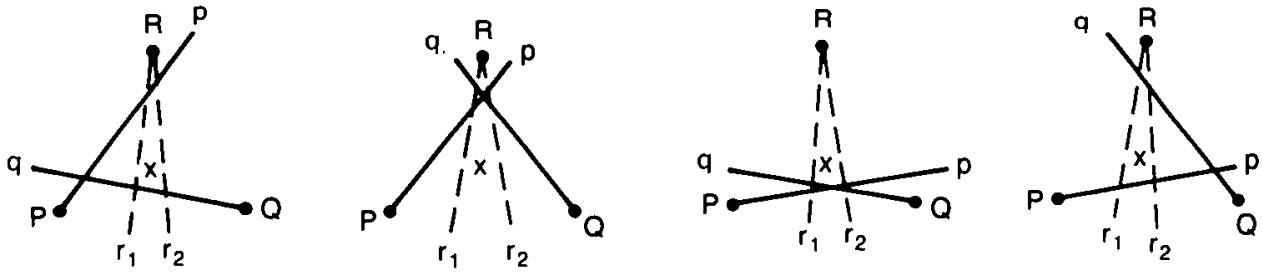

Fig. 3.

We have therefore seen two cases where the vertex and edge region probabilities are different from each other and from Mr Williams's results.

The point of the title given to this article is to emphasize that it is the cocked hat that is random, because the three bearing lines are random. The probabilities that we seek should be thought of as the probability that, for example, a random cocked hat will enclose the (fixed) true position, which may be unknown to the observer, but may, of course, be known to some outside agency. It should not be thought of as the probability that, having obtained a particular cocked hat, the true position is inside. The cocked hat is in fact a 25 percent confidence region for the true position; that is, if a large number of cocked hats is obtained, 25 percent of them can be expected to contain the true position.

Under the basic assumptions of the analysis, each bearing line has a probability $1 / 2$ that it has the true position on its left (denoted by L) and $1 / 2$ that it has the true position on its right (denoted by $R$ ). The result of the three observations can be classified into the 8 possible cases, each with probability I / 8 : LLL, LLR, LRL, LRR, RLL, RLR, RRL, $R R R$, in which each triplet indicates the side of the bearing-lines $p, q$ and $r$ that the true position lies. Under the assumption of equally likely cases, no further subdivision of results is possible. The only probabilities that can be determined without extra assumptions are events that can be described explicitly in terms of these cases.

If the true position lies inside the triangle of bearing points then the cocked hat will enclose the true position if and only if the result is LLL, or RRR. In passing it is worthy of note that if the true position is outside the triangle of bearing points then a different 
pair of results will correspond to the true position being inside the cocked hat, for example LLR and RRL as in Fig. 4, still giving, of course, the value $1 / 4$ for the probability.

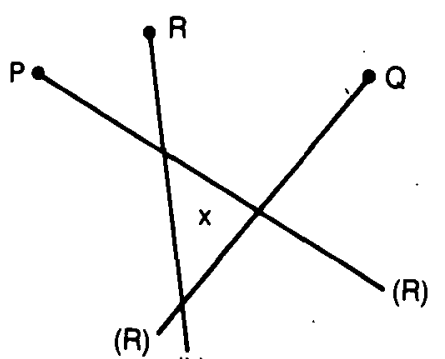

(L)

Case RRL

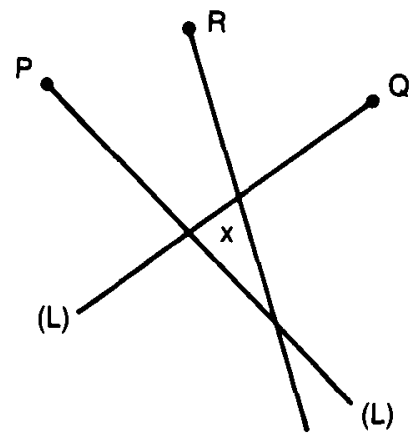

(R)

Fig. 4.

For definiteness, in subsequent work we assume that the true position is inside the triangle of bearing points.

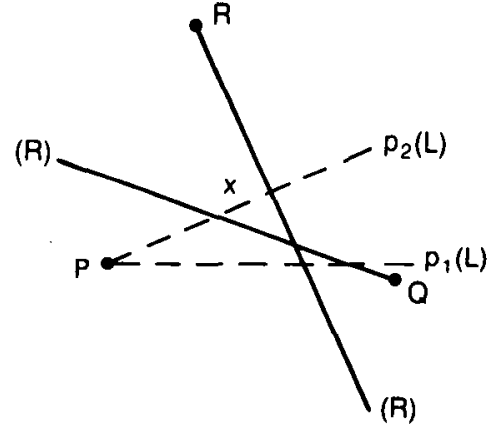

Case LRR

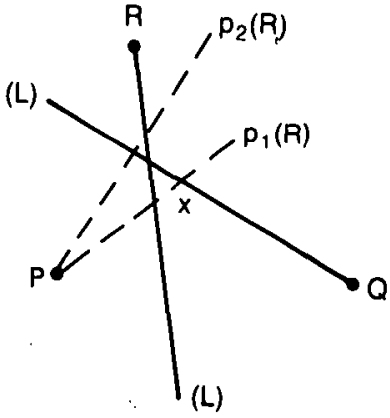

Case RLL

Fig. 5 .

From Fig. 5 it can be seen that in the case LRR, the true position is either in the $\mathrm{p}$ edge region or the qr-vertex region. This is also true for the case RLL. In fact the cases LRR and RLL together correspond exactly to the true position lying in either the p-edge or the $q r$-vertex regions, which therefore have a combined probability of $1 / 4$. Since the vertex region and the edge region cannot be separately described in the $R / L$ specification, the corresponding probabilities cannot be determined without more assumptions about the distributions of the errors.

There appear to be two different errors in Mr Williams's argument. Firstly, the total probability for the three vertex regions is not necessarily $1 / 4$ and the total probability for the three edge regions is not necessarily $1 / 2$, as demonstrated above. Secondly, in the absence of assumptions as to the equality of the distribution of the three errors, there is no reason for the three vertex regions (or the three edge regions) to have equal probabilities. The source of the first error is difficult to locate, but Mr Williams's argument seems to be based on a particular assumed result, both in the shape of the triangle and the true position being on the + side of one particular line, and so his probabilities are in some way conditional on the observed set of lines. 
REFERENCES

1 Williams, J. E. D. (1981). The cocked hat. This Journal, 44, 269.

KEY WORDS

1. Errors.

2. Statistics.

\section{J. E. D. Williams replies}

As Dr Cook states that the only assumptions permitted are the independence of errors and that each position line is equally likely to pass on either side of the true position, the early section of his note dealing with other assumptions is irrelevant. Of course, when assumptions change the probabilities may change. Dr Cook goes on: 'The probabilities that we seek should be thought of as the probability that, for example, a random cocked hat will enclose the true position. It should not be thought of as the probability that, having obtained a particular cocked hat, the true position is inside'; but the probabilities associated with the particular cocked hat which we happen to have is precisely the question I addressed. As there are later passages to which I can ascribe no meaning (how can a probability be an event?), I may only follow Dr Cook where I can.

I can follow the statement (paragraph 9): 'The result of the three observations can be classified into 8 possible cases each with a probability of $1 / 8: L L L, L L R, L R L, L R R, R L L, R L R$, $R R L, R R R$. ' In fact, one of these 'cases' is always impossible and when we see the cocked hat, we know which 'case ' it is. For the particular configuration illustrated in Dr Cook's Fig. I, the impossible 'case' is obviously LRR and each of the other 'cases' corresponds to one of the 7 'regions'. We do not assign a probability of $1 / 7$ to each region because knowledge that LRR is impossible modifies the probability of the other 'cases'.

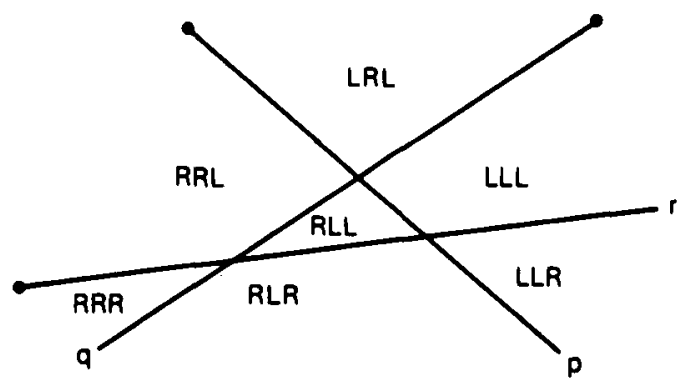

Fig. 1. LRR impossible

We know that, in this particular configuration (following Cook's notation), the true position is not to the left of $p$ or it is not to the right of $q$ or it is not to the right of $r$ because there is no LRR 'case'.

If not to the left of $p$, the position is in one of RLL, RLR, RRL or RRR.

If not to the right of $q$, the position is in one of LLL, LLR, RLL or RLR.

If not to the right of $r$, the position is in one of LLL, LRL, RLL or RRL.

Out of 12 , there are 3 ways the position can be in RLL (the triangle of the cocked 
hat), 2 ways in which it can lie in each of LLL, RLR, RRL (the edge regions), and one way in which it can lie in each of LLR, LRL, RRR (the vertex regions). QED.

In other configurations, the name of the impossible 'case' is different and the possible 'cases' correspond to different regions, but the end result is always the same; $1 / 4$ probability of being in the triangle, $1 / 6$ probability of being in each edge region, I/ 12 probability of being in each vertex region. So the argument does not depend on identifying the impossible 'case'. I make the point because it might be thought that knowledge of the shape of the cocked hat would sully the ignorance on which my argument depends to some extent. Information, like assumption, changes the probabilities.

It may seem paradoxical that, when we have a cocked hat, the probability of being on the side of any position line which contains the triangle is $2 / 3$ until we recall that there was a 50 percent probability that the position lines would give us not the cocked hat we have, but its image in which the triangle is on the other side of each position line. It is the fact that the random errors are such that they have produced the cocked hat we have and not its image which provides the information to revise the probabilities of the cases.

Dr Cook's next paragraph is entirely correct but his penultimate paragraph is wrong. We read: 'From Figure 5 it can be seen that in the case LRR, the true position is either in the

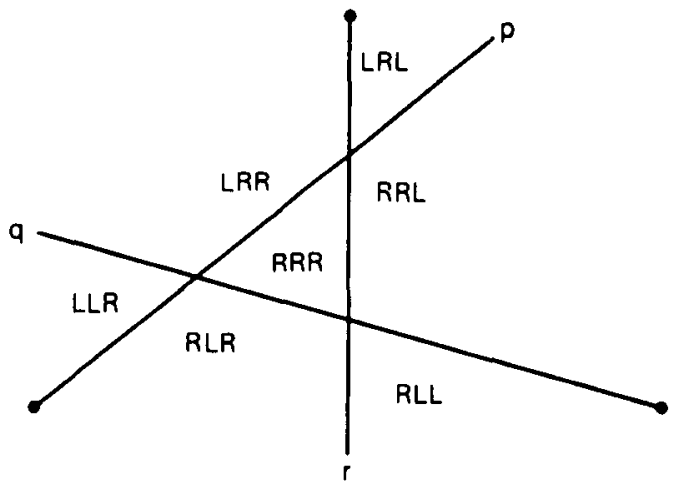

Fig. 2a. LLL impossible

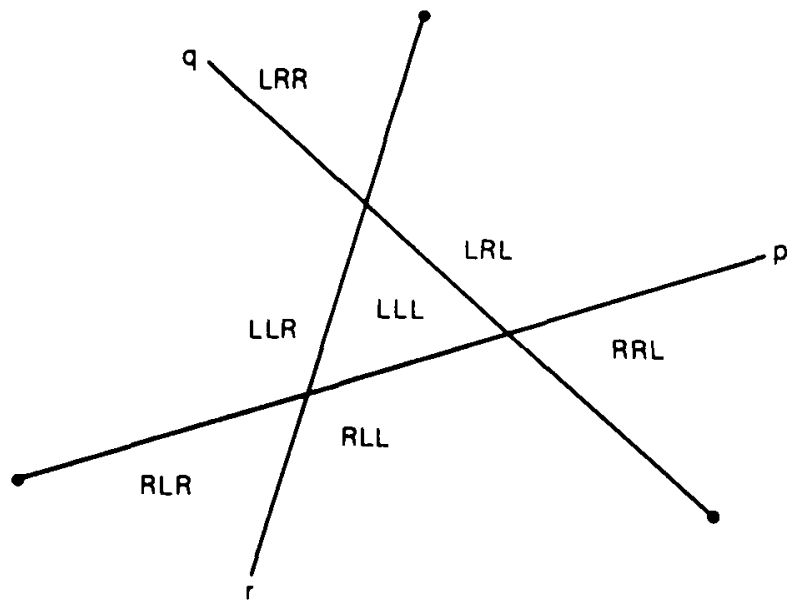

Fig. 2 b. RRR impossible 
p edge region or the qr vertex region'. In fact, in Fig. 5 , the 'case ' LRR uniquely corresponds to the $p$ edge region if the bearings are $p_{2} q r$ and to the $q r$ vertex region if the bearings are $p_{1} q r$. Of course, if you change the configuration, the correspondence between 'cases' and regions changes. Multifarious configurations arise because Dr Cook treats position lines as having a name and an arrow (e.g. $\mathrm{p}$ is away from $\mathrm{P}$, not towards it) but the difference between $p_{1} q r$ and $p_{2} q r$ is fundamental; $p_{1}$ and $p_{2}$ pass on opposite sides of the $q r$ intersection. Each is of the same configuration as the other's image.

My Fig. 2 shows the one-to-one correspondence of 'case' and region for the two configurations Dr Cook's penultimate paragraph addresses. The configuration is defined by the name of the corresponding impossible 'case'.

Concerning Dr Cook's last paragraph, I can only apologize that the extreme brevity of my little divertissement made it difficult to follow. In the days of Kalman filters, the problem is trivial, but it is not suitable for simplistic treatment.

\section{KEY WORDS}

I. Errors. 2. Statistics.

\section{EDITOR'S NOTE}

The 'cocked hat' has had a good airing in recent issues of the Journal and correspondence on the topic is now closed. 